\section{THU0647 \\ CHARACTERIZING PERSISTENCE OF IV ABATACEPT AMONG ELDERLY PATIENTS WITH RA IN THE US MEDICARE POPULATION}

Tayla Poretta, Dhaval Patil, Ying Bao, Francis Lobo. Bristol-Myers Squibb, Princeton, NJ, United States of America

Background: Rheumatoid arthritis (RA) is a chronic, systemic autoimmune disease characterized by inflammation of the joints, which can ultimately lead to cartilage damage and bone destruction. ${ }^{1}$ While early and aggressive intervention with biological treatment may alter the course of the disease and improve physical functioning, persistence to these medications varies, ranging from just 30 to $80 \%{ }^{2}$ It is important to understand the factors associated with persistence to RA treatment, as non-adherence may incur greater healthcare costs and utilization, disease progression, and the potential requirement for even more aggressive therapy in the future.

Objectives: To describe patient characteristics and baseline healthcare costs that affect persistence to intravenous (IV) abatacept, which may be supportive in evaluating the risk-benefit ratio of abatacept for treatment of RA.

Methods: Patients (aged $\geq 65$ years) with at least one inpatient or two outpatient medical claims for RA and subsequent treatment of IV abatacept from 01JUL2006 to 31DEC2016 were identified in the Truven MarketScan Medicare Supplemental database. The date of first prescription of IV abatacept was considered the index date; patients with another biologic DMARD claim on the index date were excluded. Patients were required to have 6 months continuous enrollment before and 12 months continuous enrollment following index date. Persistence was defined as the number of days from treatment initiation (index date) to first switch to another biologic DMARD, discontinuation of abatacept, or end of followup. Patients with $>6$ months of treatment were categorized as high persistence and $\leq 6$ months of treatment as low persistence. Patient characteristics were summarized by level of persistency and compared using Wilcoxon's rank sum test for continuous variables and chi-square test for categorical variables.

Results: Among 1,963 patients meeting inclusion criteria, 1,167 patients (59.4\%) were categorized as having high persistence to IV abatacept. Patient characteristics were similar at baseline between cohorts; the mean age was 74 years, $79 \%$ were female, and the mean Charlson Comorbidity Index $(\mathrm{CCl})$ score was 2.02. Compared to low persistence cohort, patients with high persistence to IV abatacept had significantly greater prior exposure to methotrexate $(44 \%$ vs $51 \%, p=0.001)$ and infliximab ( $18 \%$ vs $24 \%, p<0.001)$. Patients with high persistence to abatacept also had higher, albeit non-significant, prior use of $\mathrm{TNFi}(39 \%$ vs $41 \%$, $\mathrm{p}=0.304)$. In addition, higher persistence to abatacept was significantly associated with higher all-cause baseline medical per patient per month (PPPM) costs ( $\$ 450$ vs $\$ 475, \mathrm{p}=0.005)$ and higher RA-related baseline medical PPPM costs ( $\$ 177$ vs $\$ 231, p<0.0001)$. Patients with lower allcause baseline pharmacy PPPM costs ( $\$ 709$ vs $\$ 596, p=0.174)$ and RArelated baseline pharmacy PPPM costs ( $\$ 375$ vs $\$ 272, p=0.773$ ) had higher persistence to abatacept, although these associations were not statistically significant.

Conclusion: Among US Medicare patients with RA, surrogate markers of high disease severity, including prior methotrexate and infliximab use and higher baseline medical costs, were significantly associated with higher IV abatacept persistence. These results may assist both physicians and payers in identifying Medicare patients who benefit from abatacept and tailoring their approach accordingly to minimize the risk of nonpersistence. $^{3}$

\section{REFERENCES:}

[1] Pruijn GJ, et al. Arthritis Res Ther 2010;12:203.

[2] de Vries-Bouwstra JK, et al. Rheum Dis Clin North Am 2005;31:745-762.

${ }^{3}$ van den Bemt BJ, et al. Nat Clin Pract Rheumatol 2007;3:681.

Disclosure of Interests: Tayla Poretta Consultant for: Tayla Poretta is a consultant of Bristol-Myers Squibb Company., Dhaval Patil Shareholder of: Bristol-Myers Squibb, Employee of: Bristol-Myers Squibb, Ying Bao Employee of: Bristol-Myers Squibb, Francis Lobo Shareholder of: BristolMyers Squibb, Employee of: Bristol-Myers Squibb

DOI: 10.1136/annrheumdis-2019-eular.5044

\section{THU0648 \\ WORK DISABILITY IN A PERUVIAN COHORT OF SYSTEMIC LUPUS ERYTHEMATOSUS PATIENTS}

Cristina Reategui Sokolova ${ }^{1,2}$, Claudia Elera-Fitzcarrald ${ }^{1,3}$, Rocío Violeta Gamboa Cárdenas ${ }^{1}$, Mariela Medina ${ }^{1}$, Francisco Zevallos Miranda ${ }^{1}$, Paola Alejandra Zeña Huancas ${ }^{1}$, Victor Pimentel Quiroz ${ }^{1}$, Jorge-M Cucho-V ${ }^{1}$, Cesar Pastor Asurza ${ }^{1,4}$,

Zoila Rodriguez Bellido ${ }^{1,4}$, Risto Perich Campos ${ }^{1,4}$, Graciela S Alarcon ${ }^{5,6}$, Manuel F. Ugarte-Gil ${ }^{1}, 3 .{ }^{1}$ Hospital Nacional Guillermo Almenara Irigoyen, Lima, Peru,

${ }^{2}$ Unidad para la Generación y Síntesis de Evidencias en Salud, Universidad San Ignacio de Loyola, Lima, Peru, ${ }^{3}$ Universidad Científica del Sur, Lima, Peru, ${ }^{4}$ Universidad Nacional Mayor de San Marcos, Lima, Peru, ${ }^{5}$ University of Alabama at Birmingham, Birmingham, United States of America, ${ }^{6}$ Universidad Peruana Cayetano Heredia, Lima, Peru

Background: Work disability in patients with systemic lupus erythematosus (SLE) is common but the factors associated with it in Low and Middle Income Countries have been scarcely evaluated(1).

Objectives: To determine the prevalence of and the factors associated with work disability in SLE patients.

Methods: We studied 239 consecutive (1997 American College of Rheumatology (ACR) criteria) patients from a Peruvian SLE cohort from October 2017 to December 2018. Work disability was measured from a single self-report questionnaire. Data were collected and included sociodemographic information, clinical lupus features including disease activity [Systemic Lupus Erythematosus Disease Activity Index 2000 update (SLEDAl2K)], damage [Systemic Lupus International Collaborating Clinics/American College of Rheumatology Damage Index (SDI)], as well as Health-Related Quality of Life (LupusQoL), and medication use. Work disability was defined by patients' self-report of not being able to work because of SLE. Univariable analysis comparing those patients with work disability and those who remained working were performed with the Mann Whitney $U$ test for continuous variables and the Chi-square test for dichotomous variables. For the multivariable analyses, binary logistic regression with backward elimination was used to determine which factors remained associated with work disability.

Results: Of 239 patients, 194 patients were working at least for at least some time since diagnosis, $181(93.0 \%)$ were female, they had a mean age at diagnosis of 34.5 (12.3) years, and a mean disease duration of 11.5 (7.4) years, their mean SLEDAI was 2.53 (3.7) and their mean SDI was 1.2 (1.5). Twenty-eight patients changed their activities at work due to SLE and $51(26.6 \%)$ stopped working after their diagnosis; 21 of them $(41.1 \%)$ stopped working because of SLE. One hundred and forty-three were working at the time of the evaluation. In the multivariate analyses, those work- disabled due to SLE were more likely to have higher SDI: $\mathrm{OR}=1.650 \quad(\mathrm{Cl} 95 \%$ : 1.134-2.403) $\mathrm{p}=0.009$, and lower $\mathrm{HRQ}$ oL in two domains, planning: $\mathrm{OR}=0.975(\mathrm{Cl} 95 \%$ : 0.954-0.996) $\mathrm{p}=0.020$ and body image OR=0.977 (Cl95\%: 0.956, 0.998), $\mathrm{p}=0.032$.

Conclusion: Work disability due to SLE is associated with higher damage accrual and a poorer HRQoL.

\section{REFERENCES:}

[1] Mak A. The economic burden of systemic lupus erythematosus in Asia: the current state. Sing Lau C, editor. Lupus. 2010 Oct 14;19(12):1442-6.

Disclosure of Interests: None declared

DOI: 10.1136/annrheumdis-2019-eular.6533 